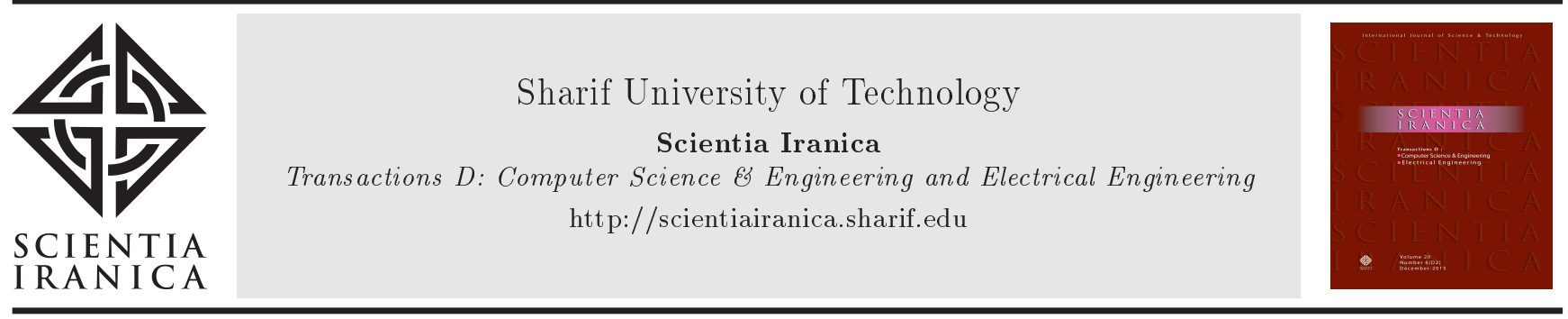

\title{
Spaser based on graphene tube
}

\section{S. Behjati Ardakani* and R. Faez}

Department of Electrical Engineering, Sharif University of Technology, Azadi Avenue, Tehran, Iran.

Received 23 January 2018; received in revised form 11 December 2018; accepted 4 February 2019

\section{KEYWORDS}

Plasmonics;

Graphene;

Spaser;

Quantum optics;

Quantum wire.

\begin{abstract}
This study proposes a structure for graphene spaser (surface plasmon amplification by stimulated emission of radiation) and develops an electrostatic model for quantizing plasmonic modes. Using this model, one can analyze any spaser consisting of graphene in the electrostatic regime. The proposed structure is investigated analytically and the spasing condition is derived. We show that spasing can occur at some frequencies where the quality factor of plasmonic modes is higher than some particular minimum values. Finally, an algorithmic design procedure is proposed by which one can design a viable structure at a given frequency. As an example, a spaser with plasmon energy of $0.1 \mathrm{eV}$ is designed.
\end{abstract}

(C) 2020 Sharif University of Technology. All rights reserved.

\section{Introduction}

Spaser, as its name suggests, is a counterpart of laser in sub-wavelength dimensions. The difference between spaser and laser is that the latter emits photons, while the former emits intense coherent Surface Plasmons (SPs). This idea was emerged after trying to overcome the main shortcoming of laser. Emission of photons in laser restricts its use in small dimensions due to the diffraction limit of light. The electromagnetic field of photons cannot be concentrated in spots that are smaller than half their wavelength, qualitatively. This is a fundamental theoretical limit and thus, cannot be circumvented. So, spaser inventors, Bergman and Stockman, suggested using another particle, instead of a photon, which does not have this theoretical constraint [1]. Their idea was to utilize the extra confined nature of SPs. SPs can confine in regions much

*. Corresponding author.

E-mail addresses: behjati@ee.sharif.ir (S. Behjati

Ardakani); faez@sharif.ir (R.Faez)

doi: $10.24200 /$ sci.2019.50322.1635 smaller than their wavelengths. In 2003, Stockman and Bergman published the first paper about the idea and introduced the word spaser to the literature [1]. Since then, many people and groups have focused on analyzing and realizing the spaser. In 2009, Noginov et al. demonstrated an experimental spaser using an aqueous solution of gold nanoparticles, each surrounded by dyedoped silica shell as a gain medium [2]. In 2010, Stockman proposed a plasmon amplifier using spaser and analyzed its equation of motion using optical Bloch equations. The author claimed that the spaser could not be analyzed classically [3]. Zhong and Li tried to analyze the spaser semi-classically in 2013 [4]. Dorfman et al. focused on the full quantum mechanical description of spaser in 2013 [5]. In 2014, Apalkov and Stockman proposed a graphene-based spaser [6]. Until now, many papers have been published covering many aspects of spaser [7-31].

Spaser, similar to laser, consists of two main parts: a medium for supporting SP modes and an active or gain medium. SPs can propagate along interface between two materials, one of which has negative dielectric constant. Metals have negative permittivity below their plasma frequencies and thus, a majority 
of papers focus on them as a medium for supporting and propagating plasmon modes. However, metals are not the ideal ones. Metal losses avoid plasmons to propagate along long distances. In this paper, we will use graphene instead of metal.

Graphene is a material that forms by a $2 \mathrm{D}$ arrangement of carbon atoms in a honeycomb lattice bonding by strong $s p^{2}$ hybridized covalent $\sigma$ bonds [32]. The $p_{z}$ electrons of carbons, lying in $\pi$ orbitals, give the graphene some extraordinary electronic properties, making it an interesting potential candidate in many applications [33-35]. The graphene electrons, near Dirac points, have a linear dispersion; thus, they behave like massless Dirac fermions. Plasmons can propagate along and confine close to graphene about an order of magnitude stronger than metals [36].

The active medium provides the energy required for initiating and maintaining the spasing process. The main factor in choosing the active medium is that which pumping mechanism we wish to use. Similar to laser, pumping method can be optical, chemical, electrical, and so forth. In our research, we are going to use electrical pumping method by utilizing a Quantum Wire (QW) as the gain medium.

In this paper, the full quantum mechanical approach is used for analyzing the structure. The most important quantity in quantum mechanics is the system's Hamiltonian. The Hamiltonian of the entire system is $H=H_{s p}+H_{a m}+H_{i n t}$, where $H_{s p}, H_{a m}$, and $H_{\text {int }}$ are SP, active medium, and interaction Hamiltonians, respectively. The individual Hamiltonian parts are quantized in the subsequent sections.

The paper is organized as follows: Section 2 introduces our proposed structure which will be used throughout the paper. Section 3 is devoted to the Hamiltonian of SP field and its quantization. Section 4 concentrates on active medium, and Section 5 is dedicated to the interaction mechanism and derivation of the spasing condition. Moreover, in this section, a procedure for designing the structure is suggested.

\section{The main structure}

Our proposed structure consists of a graphene-coated tube made of layered semiconductor heterostructure, as shown in Figure 1. The average dielectric constant of materials inside the tube is $\epsilon_{1}$. The heterostructure makes up of two layers of semiconductors with different energy gaps. The energy gap of inner rod is lower than that of outer shell so that the heterostructure forms a QW system. The inner rod plays the role of QW and the outer shell is its barrier. The graphenecoated tube is embedded in a matrix with dielectric constant $\epsilon_{2}$. Regarding the extra-confine nature of $\mathrm{SP}_{\mathrm{s}}$, it can be assumed that $\epsilon_{1}$ is equal to the outer shell dielectric constant because, roughly speaking, the

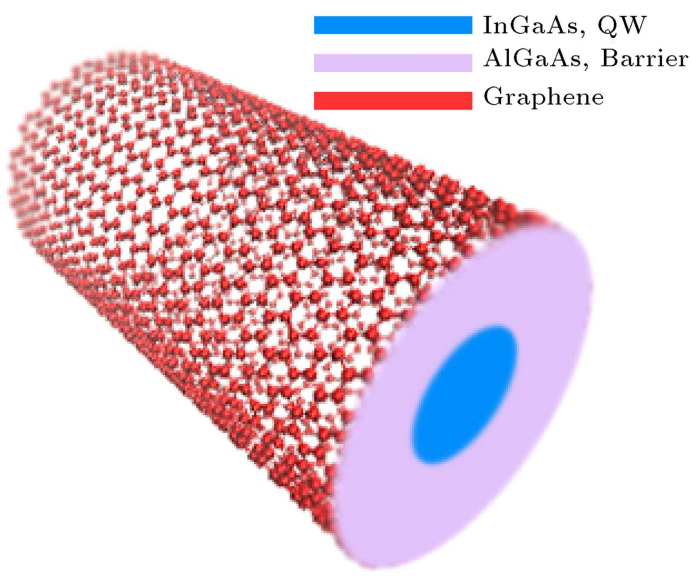

Figure 1. The proposed structure. The materials which are included in the structure are distinguished by different colors. The shown structure is embedded in a matrix of InP.

Table 1. Physical parameters of materials which are used in this paper. All the alloys are chosen such that to be lattice matched with InP at $295 \mathrm{~K}$. Dielectric constants of ternary alloys are calculated using interpolation method $[37,38]$. In this table, $\epsilon_{r}, m^{*}$, and $m_{0}$ represent dielectric constant, electron's effective mass, and electron mass, respectively.

\begin{tabular}{lcc}
\hline \multicolumn{1}{c}{ Material } & $\boldsymbol{\epsilon}_{\boldsymbol{r}}$ & $\boldsymbol{m}^{*} / \boldsymbol{m}_{\mathbf{0}}$ \\
\hline $\mathrm{InP}$ & 12.56 & 0.077 \\
$\mathrm{Al}_{0.48} \operatorname{In}_{0.52} \mathrm{As}$ & 12.46 & 0.075 \\
$\mathrm{Ga}_{0.47} \operatorname{In}_{0.53} \mathrm{As}$ & 13.60 & 0.041 \\
\hline
\end{tabular}

inner rod only senses the weak tail of SPs' field. For numerical calculations, the specific material system, $\mathrm{Al}_{0.48} \mathrm{In}_{0.52} \mathrm{As} / \mathrm{Ga}_{0.47} \mathrm{In}_{0.53} \mathrm{As}$, is used. All the materials are chosen such that they are lattice matched to the matrix, InP, at room temperature $295 \mathrm{~K}$. The required physical parameters are listed in Table 1.

The graphene tube will support plasmonic modes. The cylindrical symmetry of the configuration makes it possible to derive plasmonic modes, analytically. In addition, choosing the cylindrical structure has the advantage of dealing with fewer geometrical parameters, i.e., tube's radius, to design.

The QW is used as an active medium to provide energy for plasmons. By applying electric potential difference between graphene and QW, the electrons in QW excite. Depending on the degree of coupling strength between QW and SPs, the energy can interchange among electrons and SPs. The oscillation of energy exchange can continue steadily under some conditions. The next sections deal with finding this condition.

Throughout the paper, the tube is assumed to be infinitely long such that the edge effects can be neglected, and also its radius to be large enough 
such that size quantization effects do not influence its conductivity, significantly.

\section{SP Hamiltonian}

For quantizing the SP Hamiltonian, the orthogonal potential modes of the structure should be derived. Therefore, this section is divided into two subsections. The first subsection deals with extracting the potential modes and the second one is about writing SP Hamiltonian in the quantized form.

\subsection{Graphene tube's plasmonic modes}

Before dealing with derivation of modes, the surface conductivity of graphene is introduced. This quantity can be derived using the well-known linear response theory, Kubo formula, and Random Phase Approximation (RPA). In the low momentum regime, which means definitely not for the ultra-low doping [39] cases, the first order or local response approximation of conductivity leads to $\sigma(\omega)=\sigma_{\text {intra }}(\omega)+\sigma_{\text {inter }}(\omega)$, where:

$$
\sigma_{\text {intra }}=\frac{2 e^{2} k_{\mathrm{B}} T}{\pi \hbar^{2}} \frac{i}{\omega+i \tau^{-1}} \ln \left[2 \cosh \left(\frac{E_{\mathrm{F}}}{2 k_{\mathrm{B}} T}\right)\right],
$$

and:

$$
\begin{aligned}
\sigma_{\text {inter }}= & \frac{e^{2}}{4 \hbar} \times(\mathrm{H}(\omega / 2) \\
& \left.+\frac{4 i\left(\omega+i \tau^{-1}\right)}{\pi} \int_{0}^{\infty} \frac{[\mathrm{H}(\epsilon)-\mathrm{H}(\omega / 2)] \mathrm{d} \epsilon}{\left(\omega+i \tau^{-1}\right)^{2}-4 \epsilon^{2}}\right)
\end{aligned}
$$

with the following definition:

$$
\mathrm{H}(\epsilon)=\frac{\sinh \left(\hbar \epsilon / k_{\mathrm{B}} T\right)}{\cosh \left(E_{\mathrm{F}} / k_{\mathrm{B}} T\right)+\cosh \left(\hbar \epsilon / k_{\mathrm{B}} T\right)} .
$$

In the above relations, $e, k_{\mathrm{B}}, \hbar, T, E_{\mathrm{F}}$, and $\tau \simeq$ 0.4 ps [6] are elementary charge, Boltzmann, and reduced Planck constants, temperature, Fermi energy, and electron relaxation time, respectively. In circumstances where $\hbar \omega<2 E_{\mathrm{F}}$ and $\hbar \omega<\hbar \omega_{\text {oph }}$ (where $\hbar \omega_{\text {oph }} \simeq 0.2 \mathrm{eV}$ is optical phonon energy in graphene), Drude-like profile is a good approximation for graphene's conductivity [36]:

$$
\sigma=\frac{e^{2} E_{\mathrm{F}}}{\pi \hbar^{2}} \frac{i}{\omega+i \tau^{-1}}
$$

Out of the specified range of frequencies, two main damping channels are opened. For $\hbar \omega>2 E_{\mathrm{F}}$ the most remarkable mechanism is due to vertical Landau damping, and for $\hbar \omega>\hbar \omega_{o p h}$, optical phonon damping manifests itself. Just for simplicity of analyses, we stick to the range where these damping mechanisms are not a trouble.

After this short introduction to graphene's conductivity, we turn back to the main target, which is calculation of modes. The quasi-electrostatic approximation is utilized throughout the paper. It can be shown that this approximation describes plasmon modes quite well [40]. We will discuss the approximations in Appendix A in detail.

Considering the cylindrical symmetry of the structure, the following ansatz can be used for the electric potential:

$\phi(\mathbf{r}, t)= \begin{cases}A_{m} \mathrm{I}_{m}(k \rho) \exp i\left(k z+m \varphi-\omega_{k, m} t\right) & \rho \leq a \\ B_{m} \mathrm{~K}_{m}(k \rho) \exp i\left(k z+m \varphi-\omega_{k, m} t\right) & \rho>a\end{cases}$

where $A_{m}$ and $B_{m}$ are dependent arbitrary coefficients to be determined; $k, m, \omega_{k, m}, \mathrm{I}_{m}$, and $\mathrm{K}_{m}$ are mode indices, the corresponding frequencies, and $m$ 'th order modified Bessel functions of first and second kind, respectively. In this paper, $\rho, \phi$, and $z$ symbols are reserved for radial and angular coordinates in cylindrical coordinate system. The unit vector along any direction is denoted by adding a hat symbol above the vector associated with that direction; moreover, the hat symbol is reused for representing operators, without adding any ambiguity. Furthermore, without any loss of generality, it is assumed that graphene tube is oriented along the $z$ direction.

For the ansatz to be a valid solution, it must fulfill boundary conditions. After application of potential continuity across the boundary, $\rho=a$, the following is obtained:

$$
\frac{A_{m}}{B_{m}}=\frac{\mathrm{K}_{m}(k a)}{\mathrm{I}_{m}(k a)} .
$$

By using Ohm's law, $\mathbf{J}_{s}=\sigma_{2 D} \mathbf{E}_{t}$ (where $\mathbf{J}_{s}, \sigma_{2 D}$, and $\mathbf{E}_{t}$ are surface current density, surface conductivity, and tangential electric field, respectively) and exploiting current continuity equation, the following relation for the surface charge is derived:

$$
\begin{aligned}
\rho_{s}= & \frac{\sigma_{2 D}\left(\omega_{k, m}\right)}{i \omega_{k, m}} A_{m} \mathrm{I}_{m}(k a)\left(\frac{m^{2}}{a^{2}}+k^{2}\right) \\
& \times \exp i\left(k z+m \varphi-\omega_{k, m} t\right)+c . c .,
\end{aligned}
$$

where c.c.stands for the complex conjugate of previous terms. By using Eq. (7) and substituting it in perpendicular electric field boundary condition, another relation for coefficients is derived:

$$
\frac{B_{m}}{A_{m}}=\frac{\epsilon_{0} \epsilon_{1} k \mathrm{I}_{m}^{\prime}(k a)-\frac{\sigma_{2 \mathrm{D}}\left(\omega_{k, m}\right)}{i \omega_{k, m}}\left(\frac{m^{2}}{a^{2}}+k^{2}\right) \mathrm{I}_{m}(k a)}{\epsilon_{0} \epsilon_{2} k \mathrm{~K}_{m}^{\prime}(k a)}
$$

In the above relation, $\epsilon_{0}$ is vacuum permittivity and primes denote derivation with respect to the argument. Combining Eq. (6) with Eq. (8) leads to the dispersion relation of plasmons: 


$$
\frac{\sigma_{2 \mathrm{D}}\left(\omega_{k, m}\right)}{i \omega_{k, m} \epsilon_{0} a}=\frac{\epsilon_{1} \frac{\mathrm{I}_{m}^{\prime}(k a)}{\mathrm{I}_{m}(k a)}-\epsilon_{2} \frac{\mathrm{K}_{m}^{\prime}(k a)}{\mathrm{K}_{m}(k a)}}{\left[m^{2}+(k a)^{2}\right]} \cdot k a .
$$

Until now, no assumption has been used for surface conductivity profile. Thus, the derived dispersion relation is generalizable to any arbitrary profile of surface conductivity and is not restricted to graphene. For further simplification, we assume Drude approximation of graphene conductivity, Eq. (4), in the lossless regime, i.e., $\omega \tau \ll 1$ :

$$
\omega_{m}(k)=\sqrt{\frac{e^{2} E_{\mathrm{F}}}{\pi \hbar^{2} \epsilon_{0} a}} \frac{1}{\sqrt{\theta_{m}(k a)}},
$$

where:

$$
\theta_{m}(x) \equiv x \frac{\epsilon_{1} \frac{\mathrm{I}_{m}^{\prime}(x)}{\mathrm{I}_{m}(x)}-\epsilon_{2} \frac{\mathrm{K}_{m}^{\prime}(x)}{\mathrm{K}_{m}(x)}}{\left(m^{2}+x^{2}\right)}
$$

If we further assume $\epsilon_{1}=\epsilon_{2} \equiv \bar{\epsilon}$, then the result is more simplified:

$$
\omega_{k, m}^{2}=\frac{e^{2} E_{\mathrm{F}}}{\pi \hbar^{2} a \epsilon_{0} \bar{\epsilon}} g_{m}(x),
$$

where:

$$
g_{m}(x)=\left(m^{2}+x^{2}\right) \mathrm{I}_{m}(x) \mathrm{K}_{m}(x) .
$$

In the derivation of the above relation, the Wronskian property of the modified Bessel functions, $\mathrm{I}_{m}^{\prime}(x) \mathrm{K}_{m}(x)-\mathrm{I}_{m}(x) \mathrm{K}_{m}^{\prime}(x)=1 / x$, is utilized [41]. This result is exactly the same as the one derived from [42] by a completely different method using zeros of RPA dielectric constant. Figure 2 shows dispersion curves, Eq. (12), for some lower order modes. In drawing this figure, it is assumed that $a=100 \mathrm{~nm}$ and $E_{\mathrm{F}}=$ $0.4 \mathrm{eV}$. Figure 2(a) depicts $\hbar \omega$ normalized to Fermi energy versus $k$ normalized to Fermi wavenumber, $k_{\mathrm{F}}=$ $E_{\mathrm{F}} / \hbar v_{F}$, where $v_{F}=10^{6} \mathrm{~m} / \mathrm{s}$ is Fermi velocity, and Figure 2(b) draws normalized plasmon energy versus $k a$. From this figure, it can be seen that there are two main regions that can be discussed. For large $k / k_{\mathrm{F}}$ 's (especially in this case $k \gtrsim 0.2 k_{\mathrm{F}}$ or equivalently $\left.\hbar \omega \gtrsim 0.2 E_{\mathrm{F}}\right)$ all the modes, regardless of the value of $m$, become degenerate and have the square root feature of conventional $2 \mathrm{D}$ plasmons. This result was predictable a priori, because for large enough wavenumbers, SPs are mostly confined to the graphene and do not sense tube's radius, practically. The asymptotic form of dispersion relation, assuming Drude-like conductivity, is:

$$
\omega(k)=\sqrt{\frac{e^{2} E_{\mathrm{F}}}{2 \pi \hbar^{2} \epsilon_{0} \bar{\epsilon}}} \cdot \sqrt{k} .
$$

This result resembles that of the extended graphene. Let's look at the figure more precisely for small values of $k / k_{\mathrm{F}}$. There are two different categories of behaviors for $m=0$ and $m \neq 0$. In case of $m=0$, if the

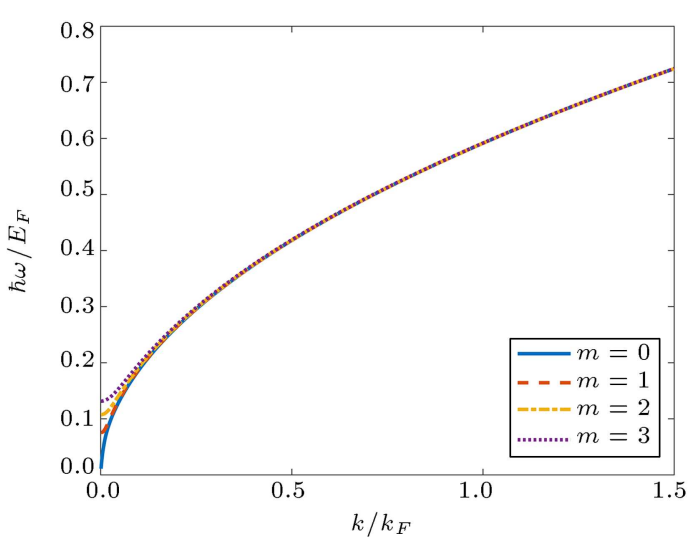

(a)

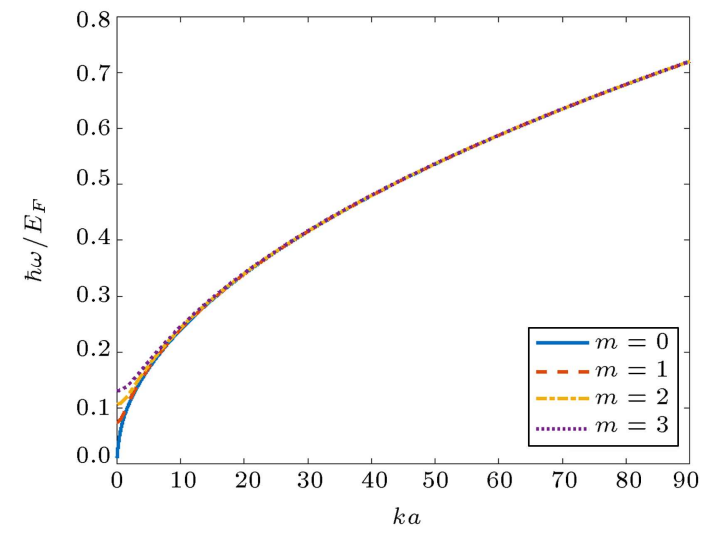

(b)

Figure 2. Plasmons dispersion for different Fermi energies. In deriving these curves, The Drude approximation is assumed: (a) Normalized plasmon energy as a function of normalized wavenumber and (b) normalized plasmon energy versus dimensionless $k a$.

These figures show that for large enough wavenumber, all the modes become degenerate.

small argument approximation of the modified Bessel functions is used, $\mathrm{I}_{0}(x) \rightarrow 1$ and $\mathrm{K}_{0}(x) \rightarrow-\ln x$, then it turns out that low momentum plasmons in graphene tube behave almost like $1 \mathrm{D}$ plasmons, i.e. $\omega_{0}(k) \simeq \omega_{\mathrm{p}} k a \sqrt{-\ln k a}$, where plasma frequency is defined as $\omega_{\mathrm{p}}=\left(e^{2} n_{0} / \epsilon m_{e}^{*}\right)^{1 / 2}$, as in 3D case except for $m_{e}^{*}$ which must be replaced by our specific definition made later in Subsection 3.2. In case of $m \neq 0$, the situation is different. It would be interesting to find a relation for $\omega_{m}(0)$. By exploiting the small argument approximation of modified Bessel functions, it can be shown that $g_{m}(0)=m / 2$ and therefore $\omega_{m}(0)=\omega_{\mathrm{p}} \sqrt{m / 2}$, roughly speaking, like the firstorder approximation of $3 \mathrm{D}$ plasmons.

It is worth mentioning that these features of dispersion are mostly due to the $2 \mathrm{D}$ nature of graphene rather than its peculiar band-structure [40], and the results are almost the same for other $2 \mathrm{D}$ materials.

It must be noted that for the Drude approximation to be applicable, $E_{\mathrm{F}}$ must lie in one of the following two regions: 


$$
\begin{gathered}
0.1<E_{\mathrm{F}}<\frac{0.08 \pi \epsilon_{0} \bar{\epsilon}}{e k}, \\
\frac{e k}{8 \pi \epsilon_{0} \bar{\epsilon}}<E_{\mathrm{F}}<0.1 .
\end{gathered}
$$

These intervals are obtained if we consider that $\hbar \omega<$ $2 E_{\mathrm{F}}$ and $\hbar \omega<\hbar \omega_{o p h} \simeq 0.2 \mathrm{eV}$. The first interval is obtained if $2 E_{\mathrm{F}}>\hbar \omega_{\text {oph }}$ and the second when $2 E_{\mathrm{F}}<$ $\hbar \omega_{o p h}$. At the above intervals, $E_{\mathrm{F}}$ is in eV unit.

Finally, the normalized potential can be written in the following form:

$$
\phi(\mathbf{r}, t)= \begin{cases}\frac{\mathrm{I}_{m}(k \rho)}{\mathrm{I}_{m}(k a)} \exp i\left(k z+m \varphi-\omega_{k, m} t\right) & \rho \leq a \\ \frac{\mathrm{K}_{m}(k \rho)}{\mathrm{K}_{m}(k a)} \exp i\left(k z+m \varphi-\omega_{k, m} t\right) & \rho>a\end{cases}
$$

The normalized potential profiles of first four lower order modes are shown in Figure 3.

The other important parameter, which will be encountered later in this work, is the Quality factor of modes. If we write $\Omega(k)=\omega(k)-i \gamma(k)$ and $\sigma_{2 D}=\sigma_{2 D}^{\prime}+i \sigma_{2 D}^{\prime \prime}$, and replace $\omega(k)$ by $\Omega(k)$ in Eq. (9), and then equating the real and imaginary parts of both sides, assuming $\gamma \ll \omega$ (which is valid for the frequency range which will be used), the Quality factor of SP modes is derived by using the definition $Q=\omega / 2 \gamma$ :

$$
Q(\omega(k))=\frac{\sigma_{2 D}^{\prime \prime}(\omega(k))}{2 \sigma_{2 D}^{\prime}(\omega(k))},
$$

Figure 4 shows the Quality factor of SP modes as a function of plasmon wavenumber and energy for
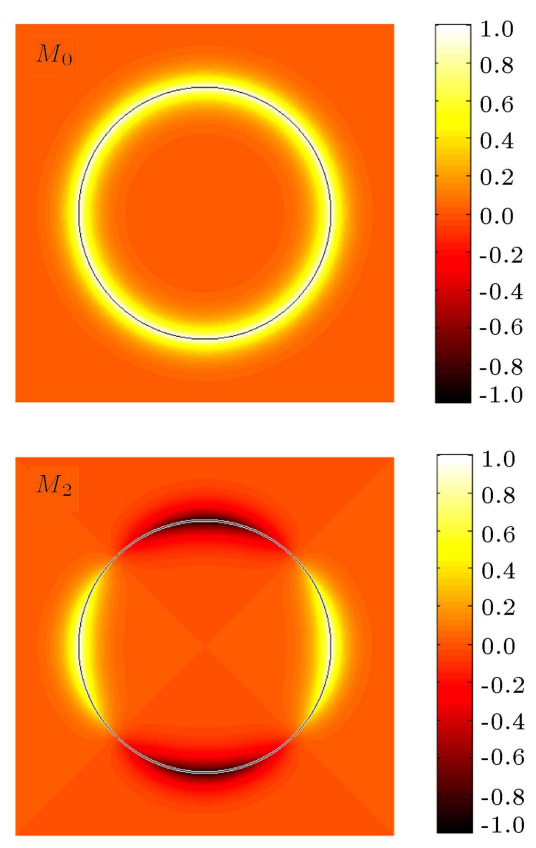

Figure 3. Cross-section view of the first four normalized potential modes so that their maximums become unity. The notation $\mathrm{M}_{m}$ is used for modes, where $m$ is the mode index defined in the paper. different values of $E_{\mathrm{F}}$ using Eq. (18). If Drude conductivity is inserted into Eq. (18), the following linear approximation can be made:

$$
Q \simeq \frac{\omega(k)}{2 \tau^{-1}}
$$

The linear behavior is apparent from Figure 4(b) in the frequency range where Drude model is more accurate, i.e., $\hbar \omega / E_{\mathrm{F}} \ll 2$.

\subsection{Quantization of SP Hamiltonian}

In the electrostatic regime, the SP Hamiltonian is composed of kinetic and potential parts, $H_{s p}=H_{k i n}+$ $H_{\text {pot }}$, where for the potential part:

$$
H_{p o t}=\frac{1}{2} \int_{S_{g}} \rho_{s} \phi \mathrm{d}^{2} r,
$$

such that $\rho_{s}$ is the surface charge density of graphene due to the existence of plasmons and $\phi$ is total electric potential. The integration runs over the graphene's surface, $S_{g}$. The kinetic part is [43]:

$$
H_{k i n}=\frac{1}{2} n_{s 0} m_{e}^{*} \int_{S_{g}}\left|\mathbf{v}_{e}\right|^{2} \mathrm{~d}^{2} r
$$

where $n_{s 0}, m_{e}^{*}$ and $\mathbf{v}_{e}$ are the surface density of electrons in equilibrium, a suggested plasmonic electron effective mass (not equal to common electron effective mass), and average drift velocity of electrons, respectively. The above kinetic Hamiltonian resembles that of 3D electron gas one. Indeed, we suggest using the same formulation for graphene, but with a modified
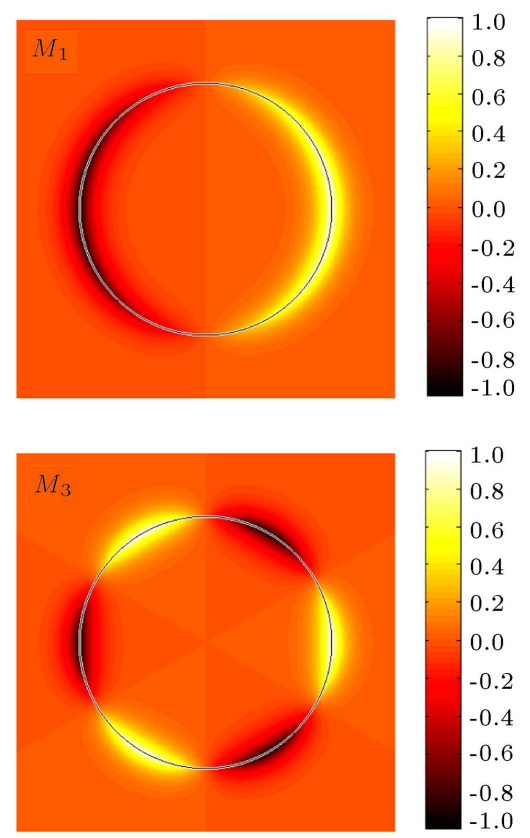


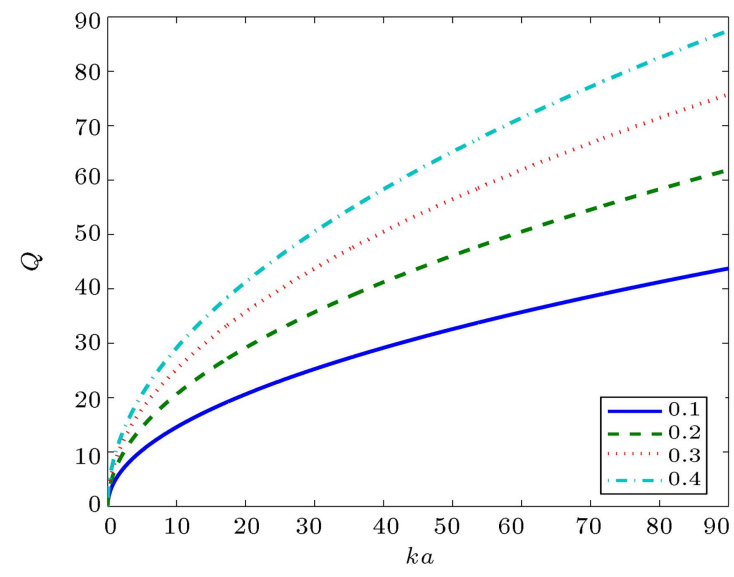

(a)

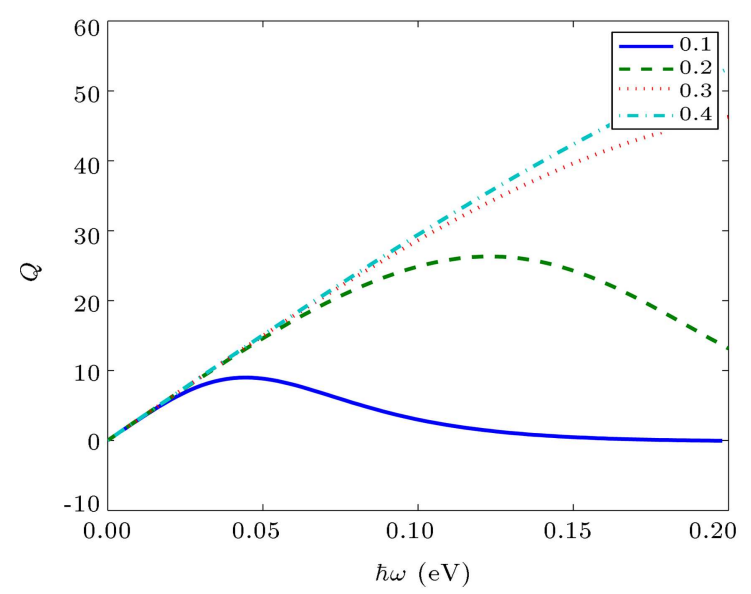

(b)

Figure 4. The quality factor of modes as a function of (a) wavenumber and (b) frequency for different Fermi energies. In Figure (b) one can see the range which linear approximation is valid.

electron effective mass. We propose finding effective mass by equating the Drude conductivity of graphene to the $3 \mathrm{D}$ electron gas one [44]:

$$
\sigma(\omega)=\frac{i \epsilon_{0} \omega_{p}^{2}}{\omega+i \gamma},
$$

where plasma frequency is defined by $\omega_{p}^{2}=e^{2} n_{0} / \epsilon_{0} m_{e}^{*}$ and $n_{0}$ is electron number density. By equating Eq. (22) to graphene conductivity, Eq. (4), one can find $m_{e}^{*}=n_{s 0} \pi \hbar^{2} / E_{\mathrm{F}}$.

For the purpose of quantizing the plasmon field, we write all the field variables in the Hamiltonian, Eq. (20) and Eq. (21), as a linear combination of plasmon modes. Therefore, the electric potential can be written in the following form:

$\phi(\mathbf{r}, t)=\sum_{k, m} C_{k, m} \phi_{k, m}(\rho) \exp i\left(k z+m \varphi-\omega_{k, m} t\right)+$ c.c.

where:

$$
\begin{aligned}
& \phi_{k, m}(\rho)=\phi_{k, m}^{+}(\rho)+\phi_{k, m}^{-}(\rho), \\
& \phi_{k, m}^{-}(\rho)=\Theta(-\rho+a) \frac{\mathrm{I}_{m}(k \rho)}{\mathrm{I}_{m}(k a)}, \\
& \phi_{k, m}^{+}(\rho)=\Theta(\rho-a) \frac{\mathrm{K}_{m}(k \rho)}{\mathrm{K}_{m}(k a)} .
\end{aligned}
$$

$C_{k, m}$ 's are expansion coefficients and $\Theta$ stands for Heaviside step function. Using this potential, surface charge density can be derived exploiting perpendicular electric field boundary condition,

$$
\begin{aligned}
\rho_{s}= & \sum_{k, m} C_{k, m} \frac{\sigma_{2 \mathrm{D}}\left(\omega_{k, m}\right)}{i \omega_{k, m}}\left(\frac{m^{2}}{a^{2}}+k^{2}\right) \\
& \times \exp i\left(k z+m \varphi-\omega_{k, m} t\right)+\text { c.c. }
\end{aligned}
$$

The only remaining quantity is drift velocity. The drift velocity can be derived using Newton's second law, $-e \mathbf{E}=m_{e}^{*} \mathrm{~d} \mathbf{v}_{e} / \mathrm{d} t$, where $\mathbf{E}=-\nabla \phi$ is electric field. After some algebra, the following result is obtained:

$$
\begin{aligned}
\mathbf{v}_{e}\left(\mathbf{r}_{\|}, t\right)= & \frac{e}{m_{e}^{*}} \sum_{k, m}\left[\frac{m}{a} \hat{\varphi}+k \hat{\mathbf{z}}\right] \frac{1}{\omega_{k, m}} C_{k, m} \\
& \times \exp i\left(k z+m \varphi-\omega_{k, m} t\right)+\text { c.c. }
\end{aligned}
$$

where $\mathbf{r}_{\|}$is the in-plane position vector. By substituting Eq. (27) and Eq. (28) into Hamiltonians, Eq. (20) and Eq. (21), and after some cumbersome algebra, the following results are found:

$$
\begin{aligned}
H_{p o t}= & \frac{A_{g}}{2} \sum_{k, m} \frac{\sigma_{2 D}\left(\omega_{k, m}\right)}{i \omega_{k, m}}\left(\frac{m^{2}}{a^{2}}+k^{2}\right) \\
& \times\left[C _ { k , m } C _ { - k , - m } \operatorname { e x p } i \left(\omega_{k, m}\right.\right. \\
& \left.\left.+\omega_{-k,-m}\right) t+C_{k, m} C_{k, m}^{*}\right]+c . c ., \\
H_{\text {kin }}= & \frac{A_{g} e^{2} n_{s 0}}{2 m_{e}^{*}} \sum_{k, m}\left(\frac{m^{2}}{a^{2}}+k^{2}\right) \\
& \times\left[\frac{-C_{k, m} C_{-k,-m}}{\omega_{k, m} \omega_{-k,-m}} \exp i\left(\omega_{k, m}+\omega_{-k,-m}\right) t\right. \\
& \left.+\frac{C_{k, m} C_{k, m}^{*}}{\omega_{k, m}^{2}}\right]+c . c .
\end{aligned}
$$

Throughout the paper, we assume that $A_{g}$ and $L$ are the hypothetical area and length of graphene tube, respectively, and $k$ and $m$ run over all possible index values. In deriving the above relations, the following orthogonality properties are exploited:

$$
\int_{-L / 2}^{L / 2} e^{i\left(k-k^{\prime}\right) z} \mathrm{~d} z=L \delta_{k, k^{\prime}}
$$




$$
\int_{0}^{2 \pi} e^{i\left(m-m^{\prime}\right) \varphi} \mathrm{d} \varphi=2 \pi \delta_{m, m^{\prime}}
$$

where $\delta$ represents Kronecker delta function. By combining Eq. (29) with Eq. (30) and assuming that $\omega_{k, m}=\omega_{-k,-m}$ the SP Hamiltonian is obtained:

$$
\begin{aligned}
H_{s p}= & \frac{A_{g}}{2} \sum_{k, m} \frac{\sigma_{2 \mathrm{D}}\left(\omega_{k, m}\right)}{i \omega_{k, m}}\left(\frac{m^{2}}{a^{2}}+k^{2}\right) \\
& \times\left(C_{k, m} C_{k, m}^{*}+C_{k, m}^{*} C_{k, m}\right) .
\end{aligned}
$$

The above Hamiltonian is analogous to harmonic oscillator's one such that by the following substitution and assuming negligible damping, $H_{s p}$ recasts to the operator form:

$$
\begin{gathered}
C_{k, m} \rightarrow \gamma_{k, m}\left(\omega_{k, m}\right) \hat{a}_{k, m}, \\
C_{k, m}^{*} \rightarrow \gamma_{k, m}\left(\omega_{k, m}\right) \hat{a}_{k, m}^{\dagger},
\end{gathered}
$$

where $\gamma_{k, m}$ is defined as follows:

$$
\gamma_{k, m}\left(\omega_{k, m}\right)=\left(\frac{\hbar \omega_{k, m}^{2}}{A_{g}\left|\sigma_{2 D}^{\prime \prime}\right|\left(\omega_{k, m}\right)}\left(m^{2} / a^{2}+k^{2}\right)\right)^{1 / 2}
$$

Using these relations, the SP Hamiltonian is simplified in the following operator form:

$$
\begin{aligned}
\hat{H}_{s p} & =\sum_{k, m} \frac{\hbar \omega_{k, m}}{2}\left(\hat{a}_{k, m}^{\dagger} \hat{a}_{k, m}+\hat{a}_{k, m} \hat{a}_{k, m}^{\dagger}\right) \\
& =\sum_{k, m} \hbar \omega_{k, m}\left(\hat{a}_{k, m}^{\dagger} \hat{a}_{k, m}+\frac{1}{2}\right),
\end{aligned}
$$

where $\hat{a}_{k m}$ and $\hat{a}_{k m}^{\dagger}$ are annihilation and creation operators of an SP in the mode $k, m$, respectively, and obey bosonic algebra [45]:

$$
\begin{aligned}
& {\left[\hat{a}_{k, m}, \hat{a}_{k^{\prime}, m^{\prime}}^{\dagger}\right]=\delta_{k, k^{\prime}} \delta_{m, m^{\prime}},} \\
& {\left[\hat{a}_{k, m}, \hat{a}_{k^{\prime}, m^{\prime}}\right]=0,} \\
& {\left[\hat{a}_{k, m}^{\dagger}, \hat{a}_{k^{\prime}, m^{\prime}}^{\dagger}\right]=0 .}
\end{aligned}
$$

By substituting Eq. (36) in Eq. (23), the electric field operator is obtained:

$$
\begin{aligned}
\hat{\mathbf{E}}(\mathbf{r}, t)= & \sum_{k, m} \gamma_{k, m}\left(\omega_{k, m}\right) \\
& \times\left[\mathbf{M}_{k, m}(\mathbf{r}) \hat{a}_{k, m}(t)+\mathbf{M}_{k, m}^{*}(\mathbf{r}) \hat{a}_{k, m}^{\dagger}(t)\right],
\end{aligned}
$$

where:

$$
\mathbf{M}_{k, m}(\mathbf{r})=\phi_{k, m}^{\prime}(\rho) \hat{\rho}+\frac{i m}{\rho} \phi_{k, m}(\rho) \hat{\varphi}+i k \phi_{k, m}(\rho) \hat{\mathbf{z}}
$$

In Eq. (41), we switched to the Heisenberg picture, where the time dependence is completely transferred to the operators.

\section{Active medium Hamiltonian}

In the present work, we propose utilizing a QW of radius $b$ and volume $V_{Q W}$ as the gain medium. For further investigation of the structure, energy levels and wavefunctions should be derived using the well-known Schrödinger equation. For the sake of simplicity and obtaining a rule of thumb, appropriate for design purposes, the infinite wall boundary condition is applied. The wavefunctions of such a structure are:

$$
\psi_{n l}(\rho, \phi, z)= \begin{cases}\frac{\exp i\left(k_{z} z+n \phi\right)}{\sqrt{V_{\mathrm{QW}} \mathrm{J}_{n+1}\left(x_{n l}\right)}} \mathrm{J}_{n}\left(\frac{x_{n l} l}{b} \rho\right) & \rho \leq b \\ 0 & \rho>b\end{cases}
$$

where $\psi_{n l}, x_{n l}$, and $k_{z}$ are $n l$ 'th eigenfunction, the l'th zero of $n$ 'th order Bessel function of the first kind, and wavenumber along the longitudinal direction, respectively. The first four lower order modes are sketched in Figure 5. The eigenenergy associated with $n l$ 'th mode is $E=E_{n l}+E_{c}$, where $E_{c}=\frac{\hbar^{2} k_{z}^{2}}{2 m_{w}^{*}}$ and energy levels $E_{n l}$ are:

$$
E_{n l}=\frac{\hbar^{2} x_{n l}^{2}}{2 m_{w}^{*} b^{2}}
$$

where $m_{w}^{*}$ is the electron's effective mass in the wire.

Maximum coupling between the QW and graphene is achieved when both are in resonance with each other, i.e., $\hbar \omega_{s p}=E_{\mathrm{e}}-E_{\mathrm{g}}$, where $\omega_{\mathrm{sp}}, E_{\mathrm{e}}$, and $E_{\mathrm{g}}$ are SP's angular frequency, and excited and ground states energy, respectively. Thus, for design purposes, $b$ maybe chosen such that the energy difference between excited and ground states coincides with plasmon energy. After some substitution and rearranging, the following result is found for QW's radius:

$$
b=\sqrt{\frac{\hbar}{2 m_{w}^{*} \omega_{s p}}\left(x_{n_{e} l_{e}}^{2}-x_{n_{g} l_{g}}^{2}\right)} .
$$

Another important quantity with a vital role in the next section is the dipole moment. The value of dipole moment represents how much the coupling strength is. It can be shown that for our structure, dipole moments only have nonzero values between states which have the same quantum number $n$. In addition, it is simple to show that dipole moment has only radial component. Dipole moment between $n l$ and $n l^{\prime}$ states is:

$$
\mathbf{d}_{n l n l^{\prime}}=2 \pi e b f_{n l l^{\prime}} \hat{\rho},
$$

where:

$$
f_{n l l^{\prime}}=\frac{1}{\mathrm{~J}_{n+1}\left(x_{n l}\right) \mathrm{J}_{n+1}\left(x_{n l^{\prime}}\right)} \int_{0}^{1} \rho^{2} \mathrm{~J}_{n}\left(x_{n l} \rho\right) \mathrm{J}_{n}\left(x_{n l^{\prime}} \rho\right) \mathrm{d} \rho
$$



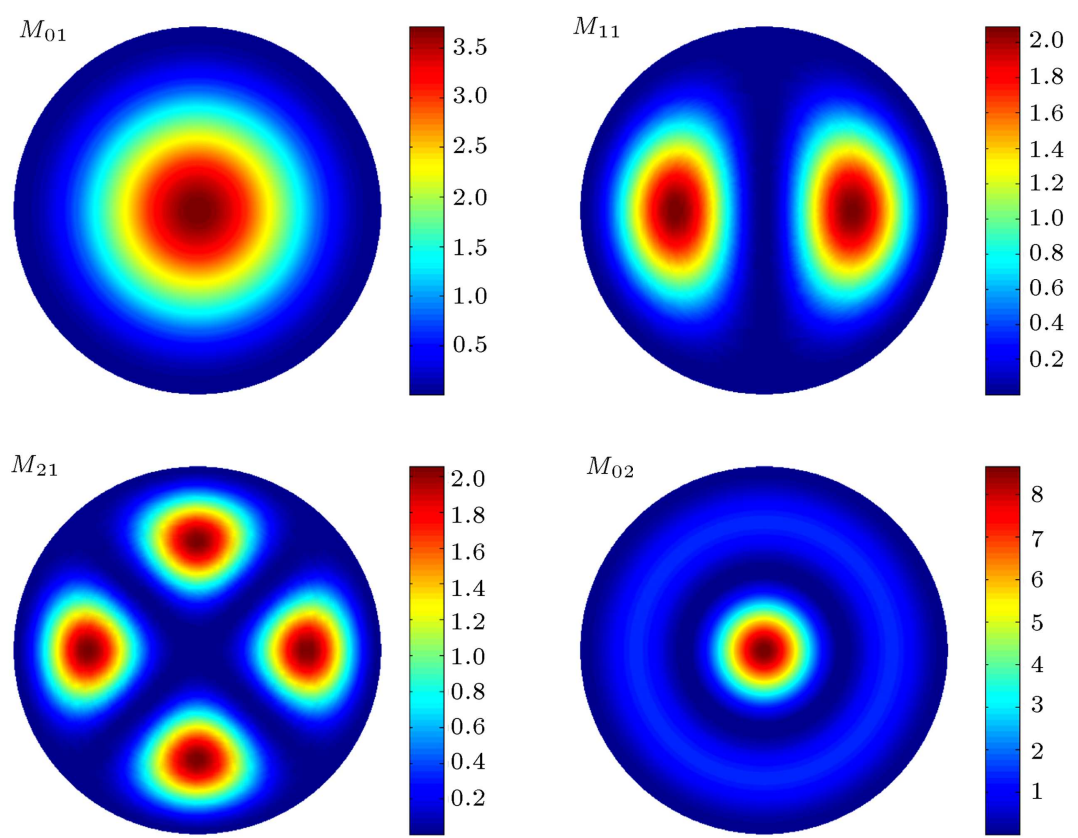

Figure 5. Cross-section view of the absolute moduli squared of the first four lower order eigenfunctions. The notation $\mathrm{M}_{n l}$ shows the mode with quantum number $n l$ discussed in the paper.

is a dimensionless number, which depends on $n, l$, and $l^{\prime}$ and it is independent of $b$. If $\psi_{01}$ and $\psi_{02}$ are considered as the states which are in resonance with a specific plasmon mode, then this number is equal to 0.09722 .

According to the spectral decomposition theorem [46], the active medium Hamiltonian, in the basis which diagonalizes itself, can be written in the following form:

$$
\hat{H}_{\mathrm{am}}=\sum_{i} E_{i} \hat{\sigma}_{i i}
$$

where $i$ is a representative of all the discrete and continuous quantum numbers and runs over all the possible states and $\hat{\sigma}_{i i}=|i\rangle\langle i|$. If we assume that the only transition, strongly coupled to the plasmon field, is $p \rightarrow q$, and further, setting the zero level of energy to the halfway between these two states, then the active medium Hamiltonian can be written in the simple form:

$$
\hat{H}_{a m}=\frac{\hbar \omega_{q p}}{2} \hat{\sigma}_{z}
$$

where the following definitions are used:

$$
\begin{aligned}
& \hat{\sigma}_{z}=|q\rangle\langle q|-| p\rangle\langle p|, \\
& \hbar \omega_{q p}=E_{q}-E_{p} .
\end{aligned}
$$

\section{Interaction Hamiltonian and spasing}

We assume that the active medium can be approximated as a dipole. Accuracy of this approximation depends on how large the multipole terms are, relative to dipole term, in the potential multipole expansion.
As a rule of thumb, the more the distance between QW and graphene, the more accurate the results will be. By using this assumption, the interaction Hamiltonian can be written as $\hat{H}_{\text {int }}=-\hat{\mathbf{d}} \cdot \hat{\mathbf{E}}$, where $\hat{\mathbf{d}}$ and $\hat{\mathbf{E}}$ are dipole moment and electric field operators, respectively. $\hat{\mathbf{d}}$ can be written down in the following form [45]:

$$
\hat{\mathbf{d}}=\mathbf{d}_{q p}\left(\hat{\sigma}_{+}+\hat{\sigma}_{-}\right)
$$

where $\mathbf{d}_{q p}$ is a dipole matrix element, associated with $p \rightarrow q$ transition, and $\hat{\sigma}_{+}=|q\rangle\langle p|$ and $\hat{\sigma}_{-}=|p\rangle\langle q|$ are rising and lowering ladder operators, respectively. After using these relations and considering energy conservation, the interaction Hamiltonian can be written as follows:

$$
\hat{H}_{i n t}=-\hbar\left[\Omega_{k m q p}\left(\mathbf{r}_{0}\right) \hat{\sigma}_{+} \hat{a}+\Omega_{k m q p}^{*}\left(\mathbf{r}_{0}\right) \hat{a}^{\dagger} \hat{\sigma}_{-}\right],
$$

where $\mathbf{r}_{0}$ is position vector of dipole and Rabi frequency, $\Omega_{k m q p}=-\hat{\mathbf{d}} \cdot \hat{\mathbf{E}} / \hbar[45]$, is written as:

$$
\Omega_{k m q p}\left(\mathbf{r}_{0}\right)=\frac{\gamma_{k, m}\left(\omega_{k, m}\right)}{\hbar} \mathbf{d}_{q p} \cdot \mathbf{M}_{k, m}\left(\mathbf{r}_{0}\right) .
$$

Taking into account that $\mathbf{d}_{q p}$ has only a component along the radial direction and substituting Eq. (42) into Eq. (54), the following result is obtained:

$$
\Omega_{k m q p}\left(\mathbf{r}_{0}\right)= \begin{cases}\frac{\gamma_{k, m}\left(\omega_{k, m}\right)}{\hbar} d_{q p} k \frac{\mathrm{I}_{m}^{\prime}\left(k \rho_{0}\right)}{\mathrm{I}_{m}(k a)} & \rho_{0}<a \\ \frac{\gamma_{k, m}\left(\omega_{k, m}\right)}{\hbar} d_{q p} k \frac{\mathrm{K}_{m}^{\prime}\left(k \rho_{0}\right)}{\mathrm{K}_{m}(k a)} & \rho_{0}>a\end{cases}
$$

where $\rho_{0}$ is QW radial position. 
The spasing condition can be written as follows $[3,6]$ :

$$
\frac{\left(\gamma_{k m}^{\prime}+\Gamma_{q p}\right)^{2}}{\left(\gamma_{k m}^{\prime}+\Gamma_{q p}\right)^{2}+\left(\omega_{q p}-\omega_{k, m}\right)^{2}} \sum_{k_{z}}\left|\Omega_{k m q p}\right|^{2} \geq \gamma_{k m}^{\prime} \Gamma_{q p}
$$

where $\Gamma_{q p}$, and $\gamma_{k m}^{\prime}$ are the damping rate of polarization and plasmon mode $k, m$, respectively, and $k_{z}$ runs over all possible transverse wavenumbers. By substituting the rabi frequency, Eq. (55), into spasing condition, Eq. (56), and assuming near resonance region, after some manipulations, we find that for spasing to be able to occur, the quality factor of SP modes should be higher than $Q_{\text {min }}$ :

$$
\begin{aligned}
Q_{\min }= & \frac{2 \pi^{2} a \hbar \epsilon_{0} \Gamma_{q p} \mathrm{Z}_{m}^{2}(k a)}{\left|d_{q p}\right|^{2} k_{\mathrm{F}} k \mathrm{Z}_{m}^{\prime 2}\left(k \rho_{0}\right)} \\
& \cdot\left[\epsilon_{1} \frac{\mathrm{I}_{m}^{\prime}(k a)}{\mathrm{I}_{m}(k a)}-\epsilon_{2} \frac{\mathrm{K}_{m}^{\prime}(k a)}{\mathrm{K}_{m}(k a)}\right],
\end{aligned}
$$

or:

$$
\begin{aligned}
Q_{\min }= & \frac{\pi a e^{2} v_{F} \Gamma_{q p} \mathrm{Z}_{m}^{2}(k a)}{\left|d_{q p}\right|^{2} \omega_{k, m}^{2} \mathrm{Z}_{m}^{\prime 2}\left(k \rho_{0}\right)} \\
& \cdot\left[\epsilon_{1} \frac{\mathrm{I}_{m}^{\prime}(k a)}{\mathrm{I}_{m}(k a)}-\epsilon_{2} \frac{\mathrm{K}_{m}^{\prime}(k a)}{\mathrm{K}_{m}(k a)}\right],
\end{aligned}
$$

where:

$$
\mathrm{Z}_{m}(x)= \begin{cases}\mathrm{I}_{m}(x) & \rho_{0}<a \\ \mathrm{~K}_{m}(x) & \rho_{0}>a\end{cases}
$$

For large values of $k$, which is the case for SPs, the condition for minimum quality factor reduces to:

$$
Q_{\min } \simeq \frac{4 \pi^{2} a \hbar \epsilon_{0} \bar{\epsilon} \Gamma_{q p}}{\left|d_{q p}\right|^{2} k_{\mathrm{F}} k},
$$

or as a function of angular frequency:

$$
Q_{\min } \simeq \frac{2 \pi a e^{2} v_{F} \Gamma_{q p}}{\left|d_{q p}\right|^{2} \omega^{2}(k)}=\frac{a v_{F} \Gamma_{q p}}{2 \pi f_{n l l^{\prime}}^{2} b^{2} \omega^{2}(k)} .
$$

Figure 6 illustrates, graphically, which frequency regions are allowable for spasing. The figure shows that for a fixed $E_{\mathrm{F}}$, there can exist an intersection point between $Q$ and $Q_{\text {min }}$ curves, which from now on we call it threshold frequency and denote it by $\omega_{t h}$. Spasing can occur for frequencies higher than $\omega_{t h}$. This dependency can be driven out analytically by combining Eq. (61) with Eq. (4):

$$
\omega_{t h}=\frac{a v_{F} \Gamma_{q p}}{\pi b^{2} f_{n l l^{\prime}}^{2} \tau}
$$

It can be seen that threshold frequency depends on the ratio of the tube's radius and cross-section area of the QW.

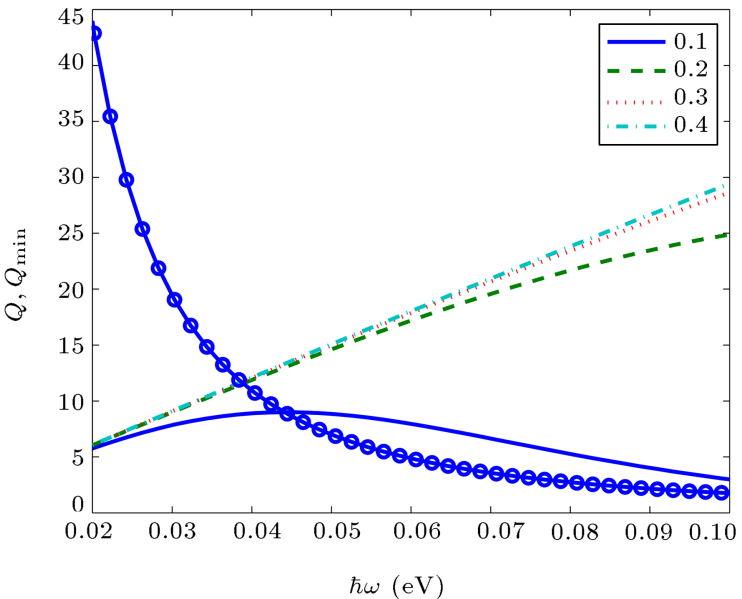

Figure 6. The solid, dashed, dotted, and dash-dotted lines show the quality factor of modes versus plasmon energy for different values of Fermi level as a parameter. Circle-marked line represents minimum required value of quality factor.

After this long discussion, we have arrived at the point where we can design a graphene tube-based spaser by using derived formulas. We intend to propose a design procedure:

1. For a given $\omega_{s p}$, calculate $b$ from Eq. (45);

2. Calculate $a$ using Eq. (62) so that $\omega_{s p}>\omega_{t h}$ or equivalently:

$$
a<\frac{\pi b^{2} f_{n l l^{\prime}}^{2} \tau}{v_{F} \Gamma_{q p}} \cdot \omega_{\mathrm{sp}}^{3} .
$$

3. Determine $E_{\mathrm{F}} k$ using Eq. (14);

4. Derive $E_{\mathrm{F}}$ and $k$ separately so that the validation ranges, Eqs. (15) or (16), are satisfied.

There exists a freedom for assigning $E_{\mathrm{F}}$ and $k$ values, separately, as long as the validation range is fulfilled. Increasing $k$ confines SPs more and more; thus, $E_{\mathrm{F}}$ can be utilized for changing spot size.

As an example, we design a spaser for $\hbar \omega_{\mathrm{sp}}=$ $0.1 \mathrm{eV}$. The calculated $b$ for this specific frequency is $15.1 \mathrm{~nm}$, the maximum value for $a$ is $1.73 \mu \mathrm{m}$ and $E_{\mathrm{F}} k=4.33 \times 10^{7}$. The maximum value for $E_{\mathrm{F}} k$ is $17.39 \times 10^{7}$ provided that we choose $E_{\mathrm{F}}>0.1 \mathrm{eV}$, Eq. (15). As long as $E_{\mathrm{F}}>0.1 \mathrm{eV}$, we can change $E_{\mathrm{F}}$ to focus SPs beam. The smallest spot is obtained where $E_{\mathrm{F}}=0.1 \mathrm{eV}$. In drawing Figure 6 , we use these values for $a$ and $b$ and further assume that $\Gamma_{q p}=3.6 \mathrm{meV}[6]$.

\section{Conclusion}

In summary, this paper suggested a structure for spasing. The structure was analyzed theoretically using full quantum mechanical approach, which treats both the field and matter quantum mechanically. In 
quantizing the Surface Plasmon (SP) field and also in writing the kinetic energy of electrons inside graphene, a special effective mass was defined. The spasing condition for the structure was derived by quantizing the Hamiltonian of the system. Finally, a design procedure was proposed and a spaser for plasmon energy of $\hbar \omega_{s p}=0.1 \mathrm{eV}$ was designed. Throughout the paper, the electrostatic approximation was used.

\section{References}

1. Bergman, D.J. and Stockman, M.I. "Surface plasmon amplification by stimulated emission of radiation: quantum generation of coherent surface plasmons in nanosystems", Physical Review Letters, 90(2), p. 027402 (2003).

2. Noginov, M., Zhu, G., Belgrave, A., Bakker, R., Shalaev, V., Narimanov, E., Stout, S., Herz, E., Suteewong, T., and Wiesner, U. "Demonstration of a spaser-based nanolaser", Nature, 460(7259), pp. 11101112 (2009).

3. Stockman, M.I. "The spaser as a nanoscale quantum generator and ultrafast amplifier", Journal of Optics, $12(2)$, p. 024004 (2010).

4. Zhong, X.L. and Li, Z.Y. "All-analytical semiclassical theory of spaser performance in a plasmonic nanocavity", Physical Review B, 88(8), p. 085101 (2013).

5. Dorfman, K.E., Jha, P.K., Voronine, D.V., Genevet, P., Capasso, F., and Scully, M.O. "Quantumcoherence-enhanced surface plasmon amplification by stimulated emission of radiation", Physical Review Letters, 111(4), p. 043601 (2013).

6. Apalkov, V. and Stockman, M.I. "Proposed graphene nanospaser", Light: Science \& Applications, 3(7), p. e191 (2014).

7. Andrianov, E., Pukhov, A., Dorofeenko, A., Vinogradov, A., and Lisyansky, A. "Forced synchronization of spaser by an external optical wave", Optics Express, 19(25), pp. 24849-24857 (2011).

8. Khurgin, J.B. and Sun, G. "Injection pumped single mode surface plasmon generators: threshold, linewidth, and coherence", Optics Express, 20(14), pp. 15309-15325 (2012).

9. Li, D. and Stockman, M.I. "Electric spaser in the extreme quantum limit", Physical Review Letters, 110(10), p. 106803 (2013).

10. Parfenyev, V.M. and Vergeles, S.S. "Quantum theory of a spaser-based nanolaser", Optics Express, 22(11), pp. 13671-13679 (2014).

11. Rupasinghe, C., Rukhlenko, I.D., and Premaratne, M. "Spaser made of graphene and carbon nanotubes", ACS Nano, 8(3), pp. 2431-2438 (2014).

12. Jayasekara, C., Premaratne, M., Stockman, M.I., and Gunapala, S.D. "Multimode analysis of highly tunable, quantum cascade powered, circular graphene spaser", Journal of Applied Physics, 118(17), p. 173101 (2015).
13. Totero Gongora, J.S., Miroshnichenko, A.E., Kivshar, Y.S., and Fratalocchi, A. "Energy equipartition and unidirectional emission in a spaser nanolaser", Laser \& Photonics Reviews, 10(3), pp. 432-440 (2016).

14. Richter, M., Gegg, M., Theuerholz, T.S., and Knorr, A. "Numerically exact solution of the many emittercavity laser problem: Application to the fully quantized spaser emission", Physical Review B, 91(3), p. 035306 (2015).

15. Meng, X., Liu, J., Kildishev, A.V., and Shalaev, V.M. "Highly directional spaser array for the red wavelength region”, Laser \& Photonics Reviews, 8(6), pp. 896-903 (2014).

16. Liu, B., Zhu, W., Gunapala, S.D., Stockman, M.I., and Premaratne, M. "Open resonator electric spaser", ACS Nano, 11(12), pp. 12573-12582 (2017).

17. Kumarapperuma, L., Premaratne, M., Jha, P.K., Stockman, M.I., and Agrawal, G.P. "Complete characterization of the spasing (11) curve of a three-level quantum coherence enhanced spaser for design optimization", Applied Physics Letters, 112(20), p. 201108 (2018).

18. Veltri, A., Chipouline, A., and Aradian, A. "Multipolar, timedynamical model for the loss compensation and lasing of a spherical plasmonic nanoparticle spaser immersed in an active gain medium", Scientific Reports, 6, p. 33018 (2016).

19. Andrianov, E., Pukhov, A., Dorofeenko, A., Vinogradov, A., and Lisyansky, A. "Spaser operation below threshold: autonomous vs. driven spasers", Optics Express, 23(17), pp. 21983-21993 (2015).

20. Ye, Y., Liu, F., Cui, K., Feng, X., Zhang, W., and Huang, Y. "Free electrons excited spaser", Optics Express, 26(24), pp. 31402-31412 (2018).

21. Shahbazyan, T.V. "Mode volume, energy transfer, and spaser threshold in plasmonic systems with gain", $A C S$ Photonics, 4(4), pp. 1003-1008 (2017).

22. Passarelli, N., Bustos-Maruún, R.A., and Coronado, E.A. "Spaser and optical amplification conditions in gold-coated active nanoparticles", The Journal of Physical Chemistry C, 120(43), pp. 24941-24949 (2016).

23. Jayasekara, C., Premaratne, M., Gunapala, S.D., and Stockman, M.I. "Mos2 spaser", Journal of Applied Physics, 119(13), p. 133101 (2016).

24. Gegg, M., Theuerholz, T.S., Knorr, A., and Richter, M. "Fully quantized spaser physics: towards exact modeling of mesoscopic CQED systems", In Ultrafast Phenomena and Nanophotonics XIX, 9361, p. $93610 Y$. International Society for Optics and Photonics (2015).

25. Petrosyan, L. and Shahbazyan, T. "Spaser quenching by offresonant plasmon modes", Physical Review B, 96(7), p. 075423 (2017). 
26. Warnakula, T., Stockman, M.I., and Premaratne, M. "Improved scheme for modeling a spaser made of identical gain elements", JOSA B, 35(6), pp. 13971407 (2018).

27. Zheng, C., Jia, T., Zhao, H., Zhang, S., Feng, D., and Sun, Z. "Low threshold tunable spaser based on multipolar fano resonances in disk-ring plasmonic nanostructures", Journal of Physics D: Applied Physics, 49(1), p. 015101 (2015).

28. Wan, M., Gu, P., Liu, W., Chen, Z., and Wang, Z. "Low threshold spaser based on deep-subwavelength spherical hyperbolic metamaterial cavities", Applied Physics Letters, 110(3), p. 031103 (2017).

29. Song, P., Wang, J.H., Zhang, M., Yang, F., Lu, H.J., Kang, B., Xu, J.J., and Chen, H.Y. "Three-level spaser for next-generation luminescent nanoprobe", Science Advances, 4(8), p. eaat0292 (2018).

30. Ardakani, S.B. and Faez, R. "Doped silicon quantum dots as sources of coherent surface plasmons", Journal of Optics, 20(12), p. 125001 (2018). URL http://stacks.iop.org/2040-8986/20/i=12/a=125001

31. Ardakani, S.B. and Faez, R., A Tunable Spherical Graphene Spaser, arXiv preprint arXiv:1712.01322 (2017).

32. Novoselov, K.S., Geim, A.K., Morozov, S., Jiang, D., Katsnelson, M., Grigorieva, I., Dubonos, S. and Firsov, A. "Two-dimensional gas of massless dirac fermions in graphene", Nature, 438(7065), pp. 197-200 (2005).

33. Majdi, M. and Fathi, D. "Graphene-based nano biosensor: Sensitivity improvement", Scientia Iranica, 24(6), pp. 3531-3535 (2017).

34. Faramarzi, V., Ahmadi, V., Ghane Golmohamadi, F., and Fotouhi, B. "A biosensor based on plasmonic wave excitation with diffractive grating structure", Scientia Iranica, 24(6), pp. 3441-3447 (2017).

35. Derakhshi, M. and Fathi, D. "Terahertz plasmonic switch based on periodic array of graphene/silicon", Scientia Iranica, 24(6), pp. 3452-3457 (2017).

36. Jablan, M., Buljan, H., and Soljačić, M. "Plasmonics in graphene at infrared frequencies", Physical Review $B, \mathbf{8 0}(24)$, p. 245435 (2009).

37. Chuang, S., Physics of Photonic Devices, Wiley Series in Pure and Applied Optics, John Wiley \& Sons (2009). URL https://books.google.com/books?id $=\mathrm{x} 5 \mathrm{Cd}_{-}$ PDf1kC.

38. Vurgaftman, I., Meyer, J., and Ram-Mohan, L. "Band parameters for iii-v compound semiconductors and their alloys", Journal of Applied Physics, 89(11), pp. 5815-5875 (2001).

39. Elias, D., Gorbachev, R., Mayorov, A., Morozov, S., Zhukov, A., Blake, P., Ponomarenko, L., Grigorieva, I., Novoselov, K., Guinea, F., et al. "Dirac cones reshaped by interaction effects in suspended graphene", Nature Physics, 7(9), p. 701 (2011).
40. Christensen, T., From Classical to Quantum Plasmonics in Three and Two Dimensions, Springer (2017).

41. Abramowitz, M. and Stegun, I.A., Handbook of Mathematical Functions: With Formulas, Graphs, and Mathematical Tables, 55, Courier Corporation (1964).

42. Longe, P. and Bose, S. "Collective excitations in metallic graphene tubules", Physical Review B, 48(24), p. 18239 (1993).

43. Arista, N.R. and Fuentes, M.A. "Interaction of charged particles with surface plasmons in cylindrical channels in solids", Physical Review B, 63(16), p. 165401 (2001).

44. Ashcroft, N. and Mermin, N., Solid State Physics, HRW International Editions, Holt, Rinehart and Winston (1976). URL https://books.google.com/books? id=1C9HAQAAIAAJ.

45. Scully, M. and Zubairy, M., Quantum Optics, Cambridge University Press (1997). URL

https://books.google.com/books?id=91kgAwAAQBAJ.

46. Hassani, S., Mathematical Physics: A Modern Introduction to Its Foundations, Springer International Publishing (2013), URL https://books.google. com/books?id=uRa4BAAAQBAJ.

47. Mikhailov, S. and Ziegler, K. "New electromagnetic mode in graphene", Physical Review Letters, 99(1), p. 016803 (2007).

48. Gao, Y., Ren, G., Zhu, B., Liu, H., Lian, Y., and Jian, S. "Analytical model for plasmon modes in graphenecoated nanowire", Optics Express, 22(20), pp. 2432224331 (2014).

49. Jalali-Mola, Z. and Jafari, S. "Electromagnetic modes from stoner enhancement: Graphene as a case study", Journal of Magnetism and Magnetic Materials, 471, pp. 220-235 (2019).

\section{Appendix A. Beyond exploited approximations}

As we mentioned in the text, electrostatic treatment can describe most features of plasmons. But, for a while, let's examine what happens if full electrodynamics is considered, qualitatively. The first and most interactive feature is the appearance of TE mode, which is not predictable by electrostatics $[47,48]$. However, it is worthwhile to mention several important notes about TE mode in graphene tube:

1. While TE and TM modes are completely distinguished in extended graphene, it is not so in graphene tube. Actually, there are no pure TE and TM modes in graphene tube, except for the case of total azimuthal symmetry, i.e., $m=0$. All the other $m \neq 0$ modes are essentially hybrid in character. $m=0$ requires some further attention. By solving Maxwell equations, it can be shown that for $m=0$, dispersion relation reduces to [48]: 


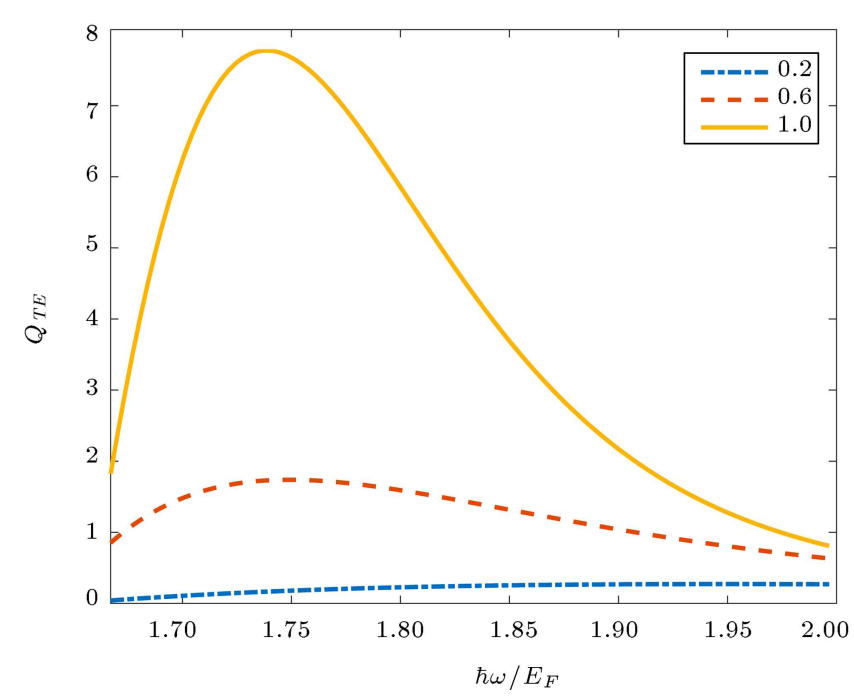

Figure A.1. Quality factor of TE mode as a function of normalized frequency in the valid range. The curves belong to different Fermi energies specified on the legend.

$$
\begin{aligned}
& \left(\frac{\epsilon_{1}}{\mu_{1}} \frac{\mathrm{I}_{1}\left(\mu_{1} a\right)}{\mathrm{I}_{0}\left(\mu_{1} a\right)}+\frac{\epsilon_{2}}{\mu_{2}} \frac{\mathrm{K}_{1}\left(\mu_{2} a\right)}{\mathrm{K}_{0}\left(\mu_{2} a\right)}-\frac{\sigma}{j \omega \epsilon_{0}}\right) \\
& \quad \times\left(\mu_{1} \frac{\mathrm{I}_{0}\left(\mu_{1} a\right)}{\mathrm{I}_{1}\left(\mu_{1} a\right)}+\mu_{2} \frac{\mathrm{K}_{0}\left(\mu_{2} a\right)}{\mathrm{K}_{1}\left(\mu_{2} a\right)}-j \omega \mu_{0} \sigma\right)=0
\end{aligned}
$$

where $\mu_{0}$ is vacuum permeability and $\mu_{i}=\left(k^{2}-\right.$ $\left.\omega^{2} \mu_{0} \epsilon_{i} \epsilon_{0}\right)^{1 / 2}$.

Apparently, TE and TM modes are decoupled. The first parenthesis on LHS led to the TM dispersion relation (for $k \gg \omega^{2} \mu_{0} \epsilon_{0}$ this equation matches Eq. (9) for $m=0$ ) and likewise, the second one to that of TE mode. So, the TE dispersion relation is:

$$
\mu_{1} \frac{\mathrm{I}_{0}\left(\mu_{1} a\right)}{\mathrm{I}_{1}\left(\mu_{1} a\right)}+\mu_{2} \frac{\mathrm{K}_{0}\left(\mu_{2} a\right)}{\mathrm{K}_{1}\left(\mu_{2} a\right)}=j \omega \mu_{0} \sigma .
$$

It is clear that TE mode exists only if $\Im \sigma<0$, which corresponds to $\hbar \omega \gtrsim 1.6671 E_{\mathrm{F}}$;

2. The Quality factor of TE mode can be found straightforwardly, using Eq. (A.2), the same as the procedure that has already been done in deriving Eq. (18). Doing some algebra leads to the following result:

$$
Q_{\mathrm{TE}}=-\frac{\sigma^{\prime \prime}}{2 \sigma^{\prime}} \quad\left(\sigma^{\prime \prime}<0\right) .
$$

Figure A.1 shows the Quality factor of TE mode as a function of frequency in the specified range, $1.6671<\hbar \omega / E_{\mathrm{F}}<2$ where TE mode exists and for different values of Fermi Energies. By comparing this figure with Figure 4(b), it is obvious that Quality factor of TE mode is an order of magnitude less than those of other TM and hybrid modes. Therefore, including the TE mode in calculations only complicates the analysis while achieving nothing more than what has been achieved by neglecting it;

3. It can be proven that TE mode exists for the cases where the absolute value of the difference between two dielectric constants surrounding graphene is quite small, which is practically hard to achieve [40]. Even if we could find two materials with very close dielectric constants, there is no guarantee that small perturbations in dielectric constants, which are common in reality, keep the criterion satisfied;

4. In case of TE mode, we have to solve full vectorial Maxwell equations. One may propose considering the TE mode by inserting it to the derived electrostatic relations, manually. Although it seems a good idea, it is absolutely wrong. Because the eigen-solutions of Laplace equation build an orthogonal basis and thus, inserting the TE mode makes the basis over-complete which is unacceptable and may lead to unphysical results.

Apart from TE mode which is a consequence of electromagnetic consideration, there exists a new hybrid mode for the undoped graphene that is absent normally. This is due to the addition of spin-flip corrections to the response function [49]. It can be shown that for the doped case, spin-flip excitations only slightly shift plasmons energy towards generically lower values [49]. However, fortunately, it is not a concern of our work because we decided not to use undoped graphene.

Finally, it can be shown that in graphene tubes, the condition $k_{\mathrm{F}} a \gg 1$ should be satisfied for the derived dispersion relation to be valid [42]. It can be checked in the final step of design procedure. For instance, considering $E_{\mathrm{F}}=0.1 \mathrm{eV}$, the condition means that $a \gg 6.9 \mathrm{~nm}$, far below our designed radius, which is in the order of micrometer.

\section{Biographies}

Sadreddin Behjati Ardakani received his BSc and MSc degrees both in Electrical and Electronics Engineering from Amirkabir University of Technology, Tehran, Iran in 2009 and 2011, respectively, and his $\mathrm{PhD}$ degree in Micro- and Nano-Electronics at Sharif University of Technology, Tehran, Iran, in 2019. His current research interests include nanooptics and optoelectronics.

Rahim Faez received his BS degree from Sharif University of Technology in 1977 and the MS and PhD degrees from UCLA in 1979 and 1985, respectively. Then, he joined Sharif University of Technology and currently he is an Associate Professor. His research interests include design and simulation of advanced semiconductor nano and quantum devices. 